\title{
Application of Kalman Filter in the Prediction of Stock Price
}

\author{
Yan Xu, Guosheng Zhang \\ Beijing Institute of Graphic Communication, Beijing, 102600, China
}

\begin{abstract}
Based on the fluctuation of the stock market and the dynamic tracking features of Kalman filter, taking stock of Changbaishan (603099) as an example, the variation process of stock price is viewed as a maneuvering system and the statespace model of stock price can be established. The forecasting result of 27 stock closing price historical data from September 22, 2014 to November 4, 2014 is given by using Kalman predictor and MATLAB computer simulation. The result shows that Kalman filter in the prediction is effective, simple and rapid.
\end{abstract} model.

Keywords-kalman filter; predict; stock price; state-space

\section{INTRODUCTION}

Over the past 20 years, the economic and financial time series has become a hot topic in economics and finance. Correct analysis and forecast of the change of financial time series can provide reliable management and decisionmaking for relevant departments and investors. Among them, the stock is a financial time series well known. The research and analysis for them will help investors making investment more accurately. The forecast of stock price need to find a suitable method because of the stock market is random and uncertain.

1940s, the classical Wiener filtering theory[1,2,3] was given by Wiener and Kolmogorov independently. It used the frequency domain method and only applied to stationary random process. In 1960, American scholar R.E. Kalman and R.S. Bucy introduced the state variables and state space concept of systems. They proposed the state space method in the time domain, and the Kalman filtering theory was created. Kalman filter was a optimal recursive algorithm which was easy real-time implementation in computer. It was suitable for dealing with multi variable systems, timevarying systems and non-stationary random processes. It can overcome the shortcomings and limitations of the classical Wiener filtering theory.

The stock market is high fluctuation and time-varying, and the Kalman filter has a good dynamic real-time tracking characteristics. Here, the stock price is analysed by using Kalman filtering theory[4,5]. As an example, the forecasting result of stock Changbaishan (603099) is given.

\section{THE KALMAN FILTER}

Consider the dynamic system

$$
\begin{aligned}
& x(t+1)=\Phi_{X}(t)+\Gamma_{W}(t) \\
& y(t)=H_{X}(t)+v(t)
\end{aligned}
$$

where $\Phi$ is the state transition matrix, $H$ is the measurement matrix, $W(t)$ is the model noise, $x(t)$ is the state vector, $y(t)$ is the measurement vector, $v(t)$ is the measurement noise, $W(t)$ and $V(t)$ are the independent white noises with zero mean, and their variance matrices are $Q$ and $R$ respectively. Then we have

\section{Lemma} as

For any two System (1) (2) have the Kalman filter

$$
\begin{gathered}
\hat{X}(t+1 \mid t+1)=\hat{x}(t+1 \mid t)+K(t+1) \mathcal{\varepsilon}(t+1) \\
\hat{X}(t+1 \mid t)=\Phi \hat{X}(t \mid t) \\
\varepsilon(t+1)=y(t+1)-H \hat{x}(t+1 \mid t) \\
K(t+1)=P(t+1 \mid t) H^{\mathrm{T}}\left[H P(t+1 \mid t) H^{\mathrm{T}}+R\right]^{-1} \\
P(t+1 \mid t)=\Phi P(t \mid t) \Phi^{\mathrm{T}}+\Gamma Q \Gamma^{\mathrm{T}} \\
P(t+1 \mid t+1)=[I-K(t+1) H] P(t+1 \mid t)
\end{gathered}
$$

with the initial values

$$
\hat{x}(0 \mid 0)=\mathrm{E} x(0)=\mu_{0},
$$$$
P(0 \mid 0)=E\left[\left(x(0)-\mu_{0}\right)\left(x(0)-\mu_{0}\right)^{\mathrm{T}}\right]=P_{0}
$$

formulae (3) (9) constituted a recursive chain, it is suitable for real time applications.

\section{THE STATE SPACE MODEL OF STOCK PRICE}

Based on the high fluctuation of the stock market and the dynamic tracking features of Kalman filter, the stock price is viewed as a maneuvering system[6], that is

$$
\begin{gathered}
x(t+1)=x(t)+T \dot{x}(t)+\frac{1}{2} a(t) T^{2} \\
\dot{x}(t+1)=\dot{x}(t)+T a(t) \\
y(t)=x(t)+v(t)
\end{gathered}
$$

where $x(t)$ and $\dot{x}(t)$ are the price and its rate of change of the stock at time $t$ respectively, $a(t)$ is the acceleration of the stock at time $t, T$ is the sampling period, $y(t)$ is the measurement, $v(t)$ is the measurement noise.

Due to the high fluctuation of the stock market, the acceleration $a(t)$ can be regarded as a white noise sequence with zero mean. Let

$$
\begin{gathered}
W(t)=\alpha(t), X(t)=\left[\begin{array}{c}
x(t) \\
\dot{x}(t)
\end{array}\right], \Phi=\left[\begin{array}{ll}
1 & T \\
0 & 1
\end{array}\right], \Gamma=\left[\begin{array}{c}
\frac{1}{2} T^{2} \\
T
\end{array}\right], \\
H=\left[\begin{array}{ll}
1 & 0
\end{array}\right]
\end{gathered}
$$

Then the system (10) (12) can be rewrited as 


$$
\begin{aligned}
& x(t+1)=\Phi_{X}(t)+\Gamma_{W}(t) \\
& y(t)=H_{X}(t)+v(t)
\end{aligned}
$$

where $w(t)$ and $v(t)$ are the independent white noises.

\section{THE APPLICATION OF KALMAN FILTER IN THE STOCK PRICE PREDICTION}

We use 27 stock closing price historical data of the stock Changbaishan (603099) from September 22, 2014 to November 4, 2014 as the experimental data, and let the sampling period of the state space model (13) (14) $T=1$, assume $Q=R=1$.

Let the state $\hat{x}(1 \mid 1)=[x(1)(x(2)-x(1)) / T]$, The forecasting result is given as Table 1 by using Kalman predictor $\hat{x}(3 \mid 2)$ of the Lemma and MATLAB computer simulation.

TABLE I. THE ACTUAL CLOSING PRICE OF THE STOCK CHANGBAISHAN (603099) AND ITS KALMAN PREDICTOR

\begin{tabular}{|c|c|c|c|c|c|c|}
\hline time & 1 & 2 & 3 & 4 & 5 & 6 \\
\hline $\begin{array}{c}\text { actual closing } \\
\text { price }\end{array}$ & 17.31 & 17.39 & 16.75 & 16.22 & 16.28 & 16.16 \\
\hline $\begin{array}{c}\text { Kalman predictor } \\
\text { initial } \\
\text { value }\end{array}$ & $\begin{array}{c}\text { initial } \\
\text { value }\end{array}$ & 17.35 & 15.99 & 15.57 & 16.22 \\
\hline absolute error & 0 & 0 & 0.60 & -0.23 & -0.71 & 0.06 \\
\hline relative error (\%) & 0 & 0 & 3.58 & -1.42 & -4.36 & 0.37 \\
\hline time & 7 & 8 & 9 & 10 & 11 & 12 \\
\hline $\begin{array}{c}\text { actual closing } \\
\text { price }\end{array}$ & 16.11 & 15.95 & 16.16 & 15.70 & 15.62 & 15.49 \\
\hline Kalman predictor & 15.92 & 15.94 & 15.67 & 16.25 & 15.12 & 15.42 \\
\hline absolute error & -0.19 & -0.01 & -0.49 & 0.55 & -0.5 & -0.07 \\
\hline relative error (\%) & -1.18 & -0.06 & -3.03 & 3.50 & -3.2 & -0.45 \\
\hline time & 13 & 14 & 15 & 16 & 17 & 18 \\
\hline actual closing price & 15.52 & 15.34 & 15.90 & 15.9 & 15.99 & 16.04 \\
\hline Kalman predictor & 15.24 & 15.43 & 15.04 & 16.34 & 15.78 & 15.96 \\
\hline absolute error & -0.28 & 0.09 & -0.86 & 0.44 & -0.21 & -0.08 \\
\hline relative error (\%) & -1.80 & 0.59 & -5.41 & 2.77 & -1.31 & -0.50 \\
\hline time & 19 & 20 & 21 & 22 & 23 & 24 \\
\hline actual closing \\
price & 16.24 & 15.58 & 14.54 & 14.84 & 15.17 & 15.10 \\
\hline Kalman predictor & 15.97 & 16.32 & 14.80 & 13.38 & 15.02 & 15.38 \\
\hline absolute error & -0.27 & 0.74 & 0.26 & -1.46 & -0.15 & 0.28 \\
\hline relative error (\%) & -1.66 & 4.75 & 1.79 & -9.84 & -0.99 & 1.85 \\
\hline time & 25 & 26 & 27 & & & \\
\hline actual closing & 15.13 & 15.37 & 15.11 & & & \\
\hline price & 14.91 & 15.04 & 15.49 & & & \\
\hline absolute error & -0.22 & -0.33 & 0.38 & & & \\
\hline relative error (\%) & -1.45 & -2.15 & 2.51 & & & \\
\hline
\end{tabular}

From Table I, it's obviously that the relative error of the stock Changbaishan's (603099) closing price is controlled under $4 \%$. Its average relative error is $2.42 \%$, but its maximum relative error reaches $9.84 \%$ (time 22 ). This phenomenon is mainly due to the abnormal stock price fluctuation for the last time and its decline range reaches $6.68 \%$. So the abnormal fluctuation of the stock price is increased by the prediction method. And at this time we also need to be combined with other methods for correction.

TABLE II. THE RELATIVE PREDICTION ERROR STATISTICS OF THE STOCK CHANGBAISHAN’S (603099) CLOSING PRICE

\begin{tabular}{|l|l|l|l|l|l|l|l|}
\hline $\begin{array}{l}\text { relative error } \\
\text { range (\%) }\end{array}$ & $0 \sim 1$ & $1 \sim 2$ & $2 \sim 3$ & $3 \sim 4$ & $4 \sim 5$ & $5 \sim 6$ & $>6$ \\
\hline number & 6 & 8 & 3 & 4 & 2 & 1 & 1 \\
\hline percentage & $24 \%$ & $32 \%$ & $12 \%$ & $16 \%$ & $8 \%$ & $4 \%$ & $4 \%$ \\
\hline
\end{tabular}

From Table II, it's obviously that the singular point's relative error more than $5 \%$ is only two. At this time we can be combined with other methods for correcting the prediction results. Such as the initial value can be selected for the smoothing result of the previous multiple times. Then the high relative prediction error caused by the abnormal stock price fluctuation can be avoided.

\section{V.CONCLUSIONS}

Based on the fluctuation of the stock market and the dynamic tracking features of Kalman filter, the variation process of stock price is viewed as a maneuvering system in this paper, and the acceleration of stock price is regarded as a white noise sequence with zero mean. Then the statespace model of stock price can be established. The forecasting result by using Kalman predictor is given. From the result of 27 historical data, we can see that the prediction method is effective, simple and rapid. Of course we still need further study and improvement if take it as a financial investment basis.

\section{ACKNOWLEDGEMENTS}

The research work was supported by the Foundation of Beijing Municipal Education Commission (KM2014100150012).

\section{REFERENCES}

[1] Deng Zi-Li, Guo Yi-Xing. Modern Time Series Analysis and Its Applications, Modelling, Filtering, Deconvolution, Predicition and Control. Beijing: Knowledge Press, 1989.

[2] Zhang Huan-Shui, Deng Zi-Li, Self-tuning optimal predictors for singular discrete stochastic linear systems. Chinese Journal of Acta Automatica Sinica, 22(1), pp.49-57, 1996.

[3] Deng Zi-Li. Optimal Filtering Theory and Applications: Modern Time Series Analysis Method. Harbin: Harbin Institute of Technology Press, 2000.

[4] Deng Zi-Li, Xu Yan, Descriptor Wiener state estimators. Journal of Automatica, 36(11), pp. 1761-1766, 2000.

[5] Xu Yan, Deng Zi-Li, Pole-assignment descriptor steady-state Kalman estimators. Chinese Journal of Acta Automatica Sinica, 29(6), pp.835-841, 2003.

[6] Tang Chun-Yan, Peng Ji-Bing, Deng Yong-Hui, Application of a realtime tracking model based on Kalman filter in the prediction of stock price. Chinese Journal of Computer Simulation, 22(9), pp.218-221, 2005 